SHEP 02-17

hep-ph/0207260

\title{
Gamma ray bursts as probes of neutrino mass, quantum gravity and dark energy
}

\author{
Sandhya Choubey and S.F. King \\ Department of Physics and Astronomy, University of Southampton, \\ Highfield, Southampton S017 1BJ, UK
}

\begin{abstract}
We calculate the time delays of neutrinos emitted in gamma ray bursts due to the effects of neutrino mass and quantum gravity using a time dependent Hubble constant which can significantly change the naive results presented hitherto in the literature for large redshifts, and gives some sensitivity to the details of dark energy. We show that the effects of neutrino mass, quantum gravity and dark energy may be disentangled by using low energy neutrinos to study neutrino mass, high energy neutrinos to study quantum gravity, and large redshifts to study dark energy. From low energy neutrinos one may obtain direct limits on neutrino masses of order $10^{-3} \mathrm{eV}$, and distinguish a neutrino mass hierarchy from an inverted mass hierarchy. From ultra-high energy neutrinos the sensitivity to the scale of quantum gravity can be pushed up to $E_{Q G} \sim 5 \times 10^{30} \mathrm{GeV}$. By studying neutrinos from GRBs at large redshifts a cosmological constant could be distinguished from quintessence.
\end{abstract}




\section{Introduction}

Gamma ray bursts (GRBs) are amongst the most distant, energetic and enigmatic astrophysical phenomena known. Understanding GRBs is arguably the most outstanding question in astronomy, and one which may be answered by a plethora of gamma ray observatories such as INTEGRAL, SWIFT and BATSE, and corresponding infrared and optical telescopes such as REM and LT [1]. It is well known that $99 \%$ of the energy of a supernova is emitted in the form of neutrinos, and therefore it is widely expected that GRBs are similarly a copious source of neutrinos which may be detected in future neutrino telescopes $[2,3,4,5]$. Within this decade it is therefore likely that GRBs will become much better understood, and their exact nature and mechanisms which drive their internal engines will be revealed. For example it may turn out that a GRB results from the core collapse of a very massive supernova to a compact rotating black hole with the energy emitted in beamed relativistic fireball jets containing copious neutrino fluxes $[6,7,8,9,10,11]$ Alternatively the GRB engine could result in the emission of beamed earth-sized cannonballs [12].

In this paper we are not concerned with detailed models of GRBs, but instead regard them as a high intensity, high energy neutrino beam with a cosmological baseline. We shall be interested in the time delay of the arrival of neutrinos. We show that the time delay may be used as a probe of three physical effects: (i) neutrino mass, (ii) quantum gravity, (iii) dark energy. The time delay due to neutrino mass has been noted earlier in [2] while effects of quantum gravity on the time of flight have been considered in $[13,14,15,16]$ for high energy photons and in $[17,18]$ for neutrinos. However none of the above papers considers the time delays due to massive neutrinos in the presence of quantum gravity, although [17] gives the dispersion relation for this case. Moreover no studies to date have calculated time delays using a formalism which correctly takes into account the time dependence of the Hubble constant due to matter and dark energy. In our study we consider the time dependence of the Hubble constant due to matter and dark energy and show that they can change the naive results by more than $100 \%$ for $z>1$. For large redshifts the results for time delays due to neutrino mass and quantum gravity are sensitive to the nature of dark energy. We show that the three effects may be disentangled by using low energy neutrinos to study neutrino mass, high energy neutrinos to study quantum gravity, and using large redshifts to study dark energy, leading to the results stated in the abstract.

For the determination of the neutrino mass, in principle one could compare the arrival time of the massive neutrinos with the arrival times of the photons emitted in the GRB, assuming them to be emitted at the same time. However this would be plausible only if the GRB were a point source in vacuo, which it is not. In any realistic GRB model the photons are trapped inside the fireball and are released much later - the exact amount of time delay being highly model dependent. Another strategy might be to compare the arrival times of the low energy neutrinos with that of the ultra-high energy ones, since (as we will show) the ultra-high energy neutrinos suffer negligible time delay due to mass. But again one would expect the low energy neutrinos to be produced thermally, as in supernovae, leading to a model dependent time delay.

However there are alternative strategies which could overcome these problems. To begin with, if the neutrinos are hierarchical in mass, $m_{1} \ll m_{2} \ll m_{3}$, then due to their mixing, neutrinos of the same energy will arrive at the detector in three bunches, corresponding to the three mass eigenstates, the arrival time only depending on their mass. We can then compare the arrival times 
of the different neutrino mass eigenstates and put limits on the neutrino mass. This represents a "clean strategy" and indeed similar arguments for constraining neutrino mass from time delays using supernova neutrinos have been used in the literature (see [19] and references therein). However we will show that with GRB neutrinos the sensitivities possible are better by orders of magnitude.

For constraining models of quantum gravity and dark energy we can use arrival times of the ultra-high energy neutrinos, which are produced over many decades of neutrino energy. We can compare the arrival times of the high energy neutrinos of different energies - which unlike the low energy thermal neutrinos are expected to be produced at almost the same time at the source - and put limits from such observations. Thus, the results in this paper do not rely on the comparison of the arrival times between photons and neutrinos, which would involve the uncertainties discussed above. However for simplicity, we shall calculate arrival time delays relative to a hypothetical low energy photon which is assumed to be emitted at the same time. This is for convenience only; in a realistic search strategy what will be important will be the comparison of time delays between neutrinos of either the same energy, or between high energy neutrinos of two different energies as discussed above.

\section{Formalism}

For a neutrino of mass $m$ and energy $E(t)$, the momentum $p(t)$ in the presence of the effects of quantum gravity (QG) with an effective energy scale $E_{Q G}$ is given to lowest order as [17]

$$
p^{2}(t) c^{2} \approx E^{2}(t)\left(1-\xi \frac{E(t)}{E_{Q G}}\right)-m^{2} c^{4}
$$

where $\xi= \pm 1$. It is worth briefly discussing the origin and reliability of the QG corrections to the dispersion relation in Eq.1. Ellis et al $[16,18]$ have shown that quantum fluctuations in space-time foam lead to a energy dependent perturbed gravitational background metric

$$
g_{i j}=\delta_{i j}, \quad g_{00}=-1, \quad g_{0 i}=u_{i} / c
$$

where $|\vec{u} / c| \sim E / E_{Q G}, E_{Q G}$ being the scale at which the quantum gravity effects set in. Such a change in the metric violates Lorentz invariance at some high scale $E_{Q G}$ and changes the dispersion relations of the particles. The linear energy dependence of such Lorentz invariance violating (LIV) terms in these models come mainly from the gravitational recoil effects. An identical form for the dispersion relation Eq. 1 is obtained by studying massive spin-1/2 fields in the framework of loop quantum gravity where the LIV breaking correction term in Eq.1 arises due to the discrete structure of space-time at Planck scale [17].

In fact the last term in Eq.1 can arise in any theory which violates Lorentz invariance. The energy dependent form of the corrections may arise from a dimension 5 LIV operator of the

form $c_{\mu \nu \lambda} \bar{\psi} \gamma_{\mu} D_{\nu} D_{\lambda} \psi$. The dimension 4 operator $c_{\mu \nu} \bar{\psi} \gamma_{\mu} D_{\nu} \psi$ would lead to an energy independent correction to the dispersion relation while the dimension 6 operator $c \bar{\psi} \gamma_{\mu} D_{\nu} D^{2} \psi$ would lead to only tiny energy independent corrections. Hence one may regard the dispersion relation in Eq.1 
as a consequence of a generic type of LIV which may arise in some particular QG theories. From the point of view of measuring neutrino masses, the QG corrections to the dispersion relation represent a possible effect which may threaten to swamp the neutrino mass effect, and therefore it is important to include the largest imaginable such effects as we do here.

Assuming Eq.1 the time dependent neutrino velocity $v(t)$ is then given to lowest order in terms of the observed neutrino energy $E_{0}$ as ${ }^{1}$

$$
v(t)=\frac{\partial E(t)}{\partial p(t)} \approx c\left(1-\frac{\epsilon_{0}^{2}}{2} \frac{a^{2}(t)}{a^{2}\left(t_{0}\right)}+\frac{3}{2} \xi \epsilon_{0}^{\prime} \frac{a\left(t_{0}\right)}{a(t)}\right)
$$

where we have expanded in $\epsilon_{0}^{2} \ll 1$ and $\epsilon_{0}^{\prime} \ll 1$ where

$$
\epsilon_{0}=\frac{m c^{2}}{E_{0}}, \quad \epsilon_{0}^{\prime}=\frac{E_{0}}{E_{Q G}} .
$$

The time-dependence of the neutrino velocity $v(t)$ arises from the expansion of the universe which redshifts the neutrino de Broglie wavelength $\lambda(t)$, and reduces their momentum and hence their velocity. The neutrino momentum $p(t)$ is related to the cosmic scale factor $a(t)$ through

$$
\frac{p(t)}{p\left(t_{0}\right)}=\frac{\lambda\left(t_{0}\right)}{\lambda(t)}=\frac{a\left(t_{0}\right)}{a(t)}
$$

Now suppose that low energy photons ${ }^{2}$ and high energy neutrinos are emitted from a GRB source at time $t_{e}$ and the low energy photons arrive on Earth at time $t_{0}$ while the neutrinos arrive at time $t_{\nu}$. The low energy photons will travel at the speed of light $c$, while the high energy neutrinos will travel with time dependent velocity $v(t)$ which may be smaller than $c$ due to the finite neutrino mass, and may be smaller or larger than $c$ due to the dispersive effects of quantum gravity. The co-moving distance $\chi$ between the GRB source and the Earth calculated in terms of the low energy photons is given by ${ }^{3}$

$$
\chi=\int_{t_{e}}^{t_{0}} \frac{c d t}{a(t)}
$$

where $a(t)$ is the cosmic scale factor. The co-moving distance $\chi$ between the GRB source and the Earth calculated using the neutrinos which travel freely with time dependent velocity $v(t)$ is

$$
\begin{aligned}
\chi & =\int_{t_{e}}^{t_{\nu}} \frac{v(t) d t}{a(t)} \\
& =\int_{t_{e}}^{t_{0}} \frac{v(t) d t}{a(t)}+\int_{t_{0}}^{t_{\nu}} \frac{v(t) d t}{a(t)}
\end{aligned}
$$

\footnotetext{
${ }^{1}$ Strictly $E_{0}$ is the neutrino energy at the time that the photons are observed on Earth $E_{0}=E\left(t_{0}\right)$, but since the neutrinos arrive a short time later this is to excellent approximation equal to the observed neutrino energy.

${ }^{2}$ As discussed in the Introduction, the time delay is defined in terms of low energy photons for convenience only. What will matter in a practical search strategy is the comparison of different time delays between neutrinos of either the same energy, or between high energy neutrinos of two different energies.

${ }^{3}$ While we have used he low energy photons for defining the co-moving distance, Eq.6 holds even for low energy massless neutrinos which travel at speed $c$.
} 
The co-moving distance in Eq.7 must be equal to that calculated using the low energy photons or low energy massless neutrinos in Eq.6.

If we now assume that the neutrino velocity and the cosmic scale factor do not change much over the time scale $t_{0}-t_{\nu}$, then equating Eq.7 to Eq.6 and using Eq.3 we find the time delay, to leading order in $\epsilon_{0}^{2}$ and $\epsilon_{0}^{\prime}$,

$$
\Delta t=t_{\nu}-t_{0} \approx \frac{\epsilon_{0}^{2}}{2} \int_{t_{e}}^{t_{0}} \frac{a(t)}{a\left(t_{0}\right)} d t-\frac{3}{2} \xi \epsilon_{0}^{\prime} \int_{t_{e}}^{t_{0}} \frac{a^{2}\left(t_{0}\right)}{a^{2}(t)} d t
$$

The time delay in Eq.8 may be expressed in terms of an integral over redshift defined as $1+z^{\prime} \equiv$ $a\left(t_{0}\right) / a(t)$, and Hubble constant defined as $H \equiv \dot{a} / a$,

$$
\Delta t \approx \frac{\epsilon_{0}^{2}}{2} I_{2}-\frac{3}{2} \xi \epsilon_{0}^{\prime} I_{-1}
$$

where $I_{n}$ are "redshift moments" of the inverse Hubble constant,

$$
I_{n}=\int_{0}^{z} \frac{d z^{\prime}}{\left(1+z^{\prime}\right)^{n} H\left(z^{\prime}\right)} .
$$

It is interesting to compare the expression for the time delay in Eq.9 to the result for the total time taken $T$ for the low energy photon or massless neutrino to travel from the GRB source to the Earth, ${ }^{4}$

$$
T=t_{0}-t_{e}=I_{1}
$$

It is also interesting to compare to the result for the proper distance $D$ of the GRB source to the Earth at the photon arrival time, ${ }^{5}$

$$
D=a\left(t_{0}\right) \chi=c I_{0} .
$$

The upper limit of the integrals in Eq.10 z represents the redshift of the GRB source, where $1+z=a\left(t_{0}\right) / a\left(t_{e}\right)$, and the Hubble constant $H(z)$ for a flat universe is given by

$$
H^{2}(z) / H_{0}^{2}=\Omega_{M}(1+z)^{3}+\Omega_{D E} e^{I(z)}
$$

where

$$
I(z)=3 \int_{0}^{z}\left(1+w\left(z^{\prime}\right)\right) \frac{d z^{\prime}}{1+z^{\prime}}
$$

where the equation of state for the dark energy $w$, defined as the ratio of its pressure to its density $w \equiv p / \rho$, in quintessence models has a redshift dependence. For constant $w$ we have

$$
H^{2}(z) / H_{0}^{2}=\Omega_{M}(1+z)^{3}+\Omega_{D E}(1+z)^{3(1+w)}
$$

\footnotetext{
${ }^{4}$ The time $T$ represents how far back in time the GRB took place. As $z \rightarrow \infty, T \rightarrow T_{0}$ where $T_{0}$ is the age of the universe.

${ }^{5} D$ represents the actual distance of the GRB from the Earth as measured now. As $z \rightarrow \infty, D \rightarrow H_{D}$ where $H_{D}$ is the horizon distance that light could have travelled since the beginning of the universe, which represents the size of the observable universe.
} 


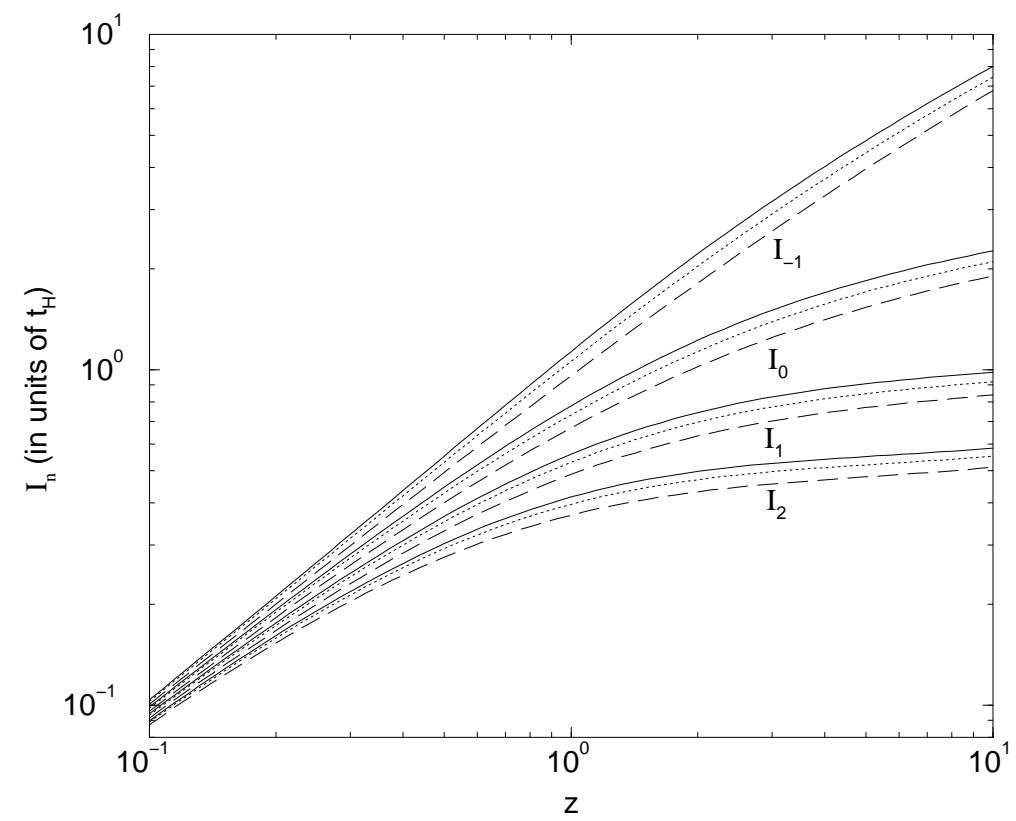

Figure 1: The integrals $I_{n}$ in units of the Hubble time $t_{H} \approx 14$ billion years as a function of the red shift $\mathrm{z}$ for three different cosmological models: CONST (solid), SUGRA quintessence (dots), INV quintessence (dashes). $I_{0}$ is the distance of the GRB in light years, $I_{1}$ is the date (years B.C.) that the GRB exploded, $I_{2}$ is used to calculate the time delay $\Delta t$ due to neutrino mass, and $I_{-1}$ is used to calculate the time difference due to quantum gravity effects as discussed in the text.

and for a cosmological constant $\Lambda$ with $w=-1$ and $\Omega_{D E}=\Omega_{\Lambda}$ this reduces to,

$$
H^{2}(z) / H_{0}^{2}=\Omega_{M}(1+z)^{3}+\Omega_{\Lambda} .
$$

The present day ratio of matter $(\mathrm{M})$ density to critical density is $\Omega_{M} \approx 0.3$, the present day ratio of dark energy (DE) density to critical density is $\Omega_{D E} \approx 0.7$ and the Hubble constant is $H_{0} \approx 70 \mathrm{kms}^{-1} \mathrm{Mpc}^{-1}$.

We shall consider three different cosmological models which were recently parametrised in [20]: cosmological constant (CONST) with potential $V \sim \Lambda^{4}$; supergravity (SUGRA) inspired quintessence field (Q) model with potential $V(Q) \sim 1 / Q^{11} e^{Q^{2} / 2}$; inverse (INV) power law quintessence field (Q) model with potential $V(Q) \sim 1 / Q^{6}$. The equation of state $w(z)$ for the SUGRA and INV models does not have a simple analytic form, but it may be parametrised as discussed in [20], and we shall use the parametrisation given there in this paper. Each of these models has $w(z)$ which varies with $z$ in such a way as to lead to a "tracking" behavior. This implies in particular that the value of the dark energy density remains of the same order as the matter density for large redshifts. By comparison the ratio of the cosmological constant energy density to the matter density falls as $1 /(1+z)^{3}$ and rapidly becomes negligible at large redshifts.

In Figure 1 we calculate $I_{n}$ in Eq.10 for $n=-1,0,1,2$ in units of the Hubble time $t_{H}=1 / H_{0} \approx$ $14 \mathrm{Gyr}$ as a function of redshift $z$ for the three different cosmological models CONST, SUGRA, INV defined above. The physically relevant quantities $D, T$ and $\Delta t$ are simply related to $I_{0}, I_{1}$, 
$I_{2}$ and $I_{-1}$ as shown in Eq.12,11,9. Figure 1 shows that as $n$ increases $I_{n}$ becomes increasingly insensitive to redshift (flatter curves) and to the particular cosmological model (closer spacing between solid, dotted and dashed curves) at large redshift. Both effects can be simply understood as being due to the factor of $\left(1+z^{\prime}\right)^{n}$ in the denominator which tends to suppress the contributions from the higher redshift parts of the integration region for larger $n$. The difference between the curves for $I_{0}$ and those for $I_{-1}, I_{2}$ in Figure 1 represents the error that would be made if the time delay were calculated using the naive formula

$$
\Delta t^{\text {naive }} \approx\left(\frac{\epsilon_{0}^{2}}{2}-\frac{3}{2} \xi \epsilon_{0}^{\prime}\right) \frac{D}{c} \approx\left(\frac{\epsilon_{0}^{2}}{2}-\frac{3}{2} \xi \epsilon_{0}^{\prime}\right) I_{0}
$$

which ignores the effect of neutrino redshift as has been done up till now in the literature rather than the correct formula for $\Delta t$ in Eq.9. For $z=1$ the error incurred by using the naive formula can clearly be seen to be of order $100 \%$, with the error rapidly growing for larger redshifts. It is also clear from Figure 1 that there is some sensitivity to the nature of dark energy for large redshifts.

Finally it is interesting to consider the matter dominated limit of our time delay result corresponding to $\Omega_{M}=1, \Omega_{D E}=0$. In this limit the expression for the time delay in Eq.9 reduces to

$$
\Delta t^{\text {matter }} \approx \frac{\epsilon_{0}^{2}}{5 H_{0}}\left[1-\frac{1}{(1+z)^{5 / 2}}\right]-\frac{3 \xi \epsilon_{0}^{\prime}}{H_{0}}\left[(1+z)^{1 / 2}-1\right]
$$

Time delays due to quantum gravity effects in the matter dominated limit were also considered in [16]. However the result quoted there corresponds to the second term in Eq.17 rather than the second term in Eq.18 which correctly takes into account the expansion of the universe.

\section{Results}

The results in this section are based on the full formula for $\Delta t$ in Eq.9, using the Hubble constant for a flat universe calculated using Eq.13 for the different dark energy models.

Figure 2 shows the time delays of the neutrinos against observed neutrino energy $E_{0}$ for a fixed GRB redshift of $z=1$ and assuming the cosmological constant model. In each panel the downward sloping dotted lines give the time delay due to the effect of neutrino mass which in the upper panels is chosen to be $m=0.05 \mathrm{eV}$ corresponding to the "atmospheric neutrino mass" defined as the square root of the atmospheric mass squared splitting [21], and in the lower panels we take $m=0.005 \mathrm{eV}$ corresponding to the "solar neutrino mass" which is the square root of the large mixing angle solar mass squared splitting [22, 23]. To probe neutrino mass corresponding to the solar scale the detector has to observe neutrinos with $E_{0} \sim$ few $10 \mathrm{MeV}$ with time sensitivity of a few milliseconds. The time delays corresponding to the atmospheric scale are higher and should be easier to detect in the planned neutrino telescopes.

The upward sloping dashed lines show the gravitationally induced time delay of the neutrinos. The upper panels give the time delay when the quantum gravity energy scale corresponds to the Planck scale. The lower panels are for the case where the quantum gravity corrections become 
important at $10^{22} \mathrm{GeV}$. The left panels have $\xi=+1$ corresponding to a negative time delay due to quantum gravity which tends to cancel with the positive time delay from the effect of neutrino mass. For this case the higher energy neutrinos arrive earlier. In the figure we have plotted the absolute value of the difference between their arrival times. The right panels have $\xi=-1$ corresponding to a positive time delay due to quantum gravity which reinforces the positive time delay from the effect of neutrino mass. For both the cases we observe that the quantum gavity effects become very important for neutrinos arriving with energies greater than a few GeV. The ultra high energy neutrinos travelling cosmological distances can put severe constraints on $E_{Q G}$. Note that the arguments in [18] suggest that fermions travel more slowly than $c$ and hence that $\xi=-1$.

The solid lines in the figure show the time delay of the neutrinos due to the sum of the dotted and dashed lines when both the effects are present. For the case where $\xi=+1$ the two effects can cancel each other when from Eq.9 we have $\Delta t=0$ which occurs at an energy

$$
E_{0}^{\prime}=\left(\frac{m^{2} E_{Q G}}{3} \frac{I_{2}}{I_{-1}}\right)^{\frac{1}{3}} .
$$

For $\xi=-1$ even though the two effects reinforce each other, we see from Eq.9 and fig. 2 that the minima in $\Delta t$ comes at exactly the same energy.

Figure 2 shows that time delay due to neutrino mass is important for the lower energy neutrinos with $E_{0}<E_{0}^{\prime}$, while the time delay due to quantum gravity effects is important for the higher energy neutrinos with $E_{0}>E_{0}^{\prime}$. This enables the two effects to be disentangled and treated separately.

\subsection{Neutrino Mass Limits from Low Energy Neutrinos}

In this section we shall focus on neutrino events with energy $E_{0}<E_{0}^{\prime}$ in order to put limits on $m$. This implies that neutrinos with energies less than $\sim 100 \mathrm{MeV}$ can be effectively used to study their mass. Thermal neutrinos with energies between $10-100 \mathrm{MeV}$ are expected to be produced in GRBs [2, 11]. Thermal neutrinos, coming from GRBs which are energetic enough, which are not very far away and which are probably beamed towards the Earth, should be detectable in the future $\mathrm{km}^{2}$ ice detectors like IceCube. These lowest energy neutrinos are expected to be detected mainly via $\bar{\nu}_{e}+p \rightarrow n+e^{+}$where the positrons lead to an increase in the low PMT noise. This detection method which forms part of a Supernova Early Warning System may also be used to detect low energy neutrinos from GRB's $[2,11]$. However the determination of neutrino mass using the time delay techniques requires sensitivity to both arrival times as well as energy of the incoming neutrino. For a detector like IceCube since the inter-PMT distance is large the energy resolution is expected to be poor, particularly for the lower energy neutrino events. However energy resolution of the proposed megaton Super-Kamiokande type water Cerenkov detectors such as Hyper-K, UNO and TITAND should be good and can be effectively used for putting direct limits on neutrino mass. We next calculate the number of events that these detectors would observe from a GRB event.

One can estimate the number of thermal neutrinos emitted in a typical GRB by the following argument $[2,11]$. Assume that the energy of the GRB in photons is $E_{\gamma}$ and the radius of the GRB 


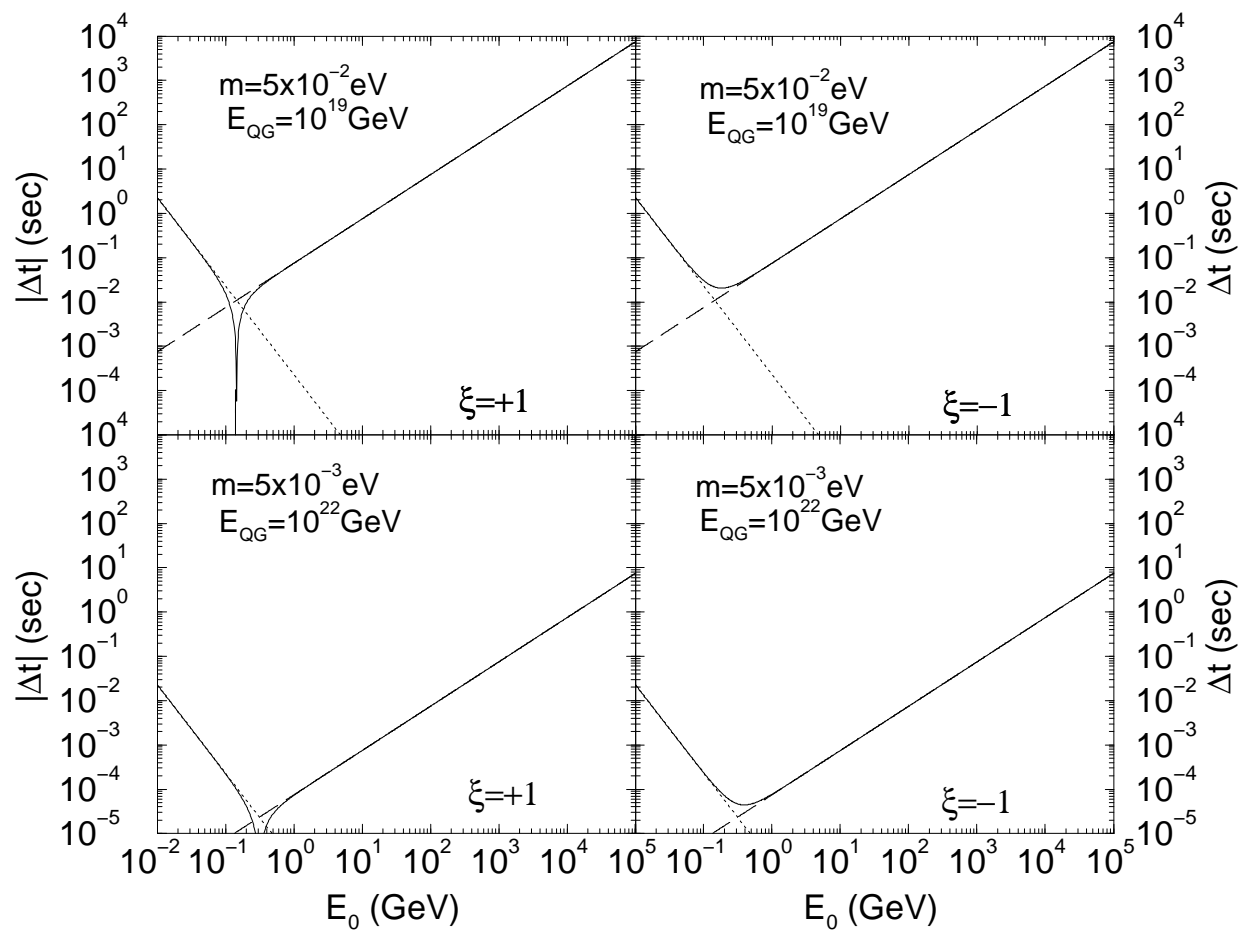

Figure 2: Time delay $\Delta t$ due to the effect of both neutrino mass and quantum gravity against observed neutrino energy $E_{0}$ for $z \sim 1$ for the cosmological constant model.

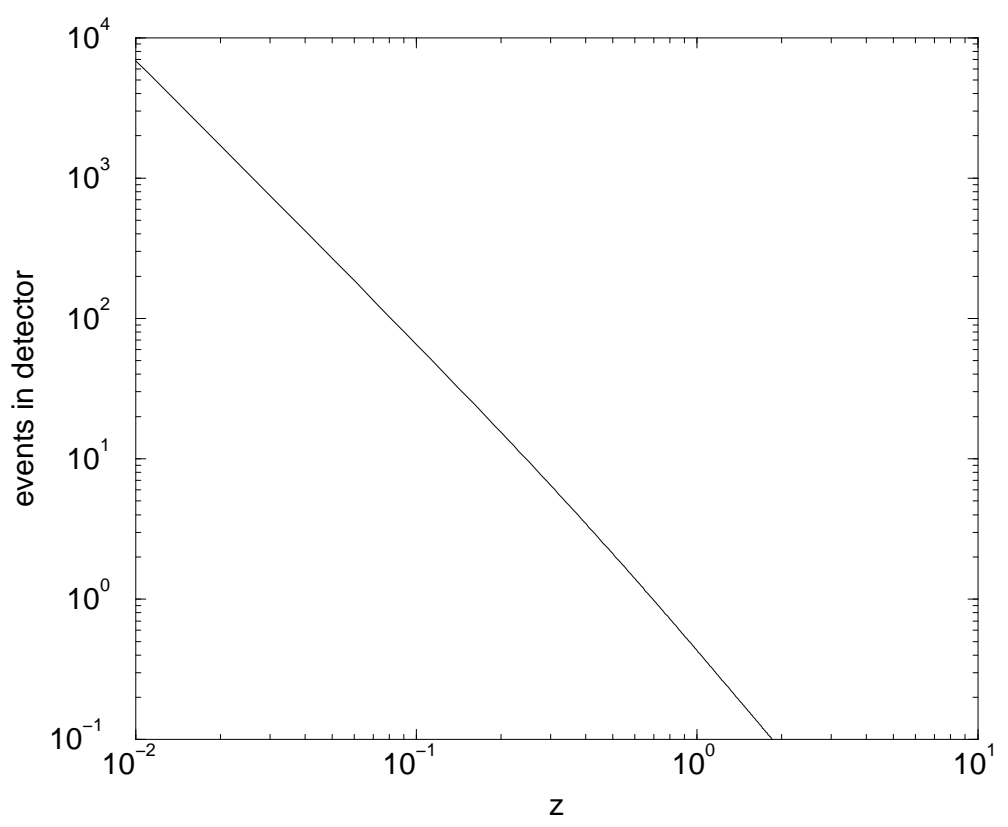

Figure 3: Number of events expected from thermal neutrinos in a one megaton water cerenkov detector as a function of the GRB redshift $z$. 
fireball is $R$, then the photon temperature $T_{\gamma}$ is given by $T_{\gamma}=\left(2 E_{\gamma} / \sigma V h_{\gamma}\right)^{1 / 4}$, where $V=\frac{4}{3} \pi R^{3}$, $\sigma$ is the Stefan-Boltzmann constant and $h_{\gamma}=2$ is the number of degrees of freedom. Similarly the neutrino temperature $T_{\nu}$ is given by $T_{\nu}=\left(2 E_{\nu} / \sigma V h_{\nu}\right)^{1 / 4}$, where the number of degrees of freedom for the neutrinos is $h_{\nu}=2 \times 3 \times \frac{7}{8}$ and $E_{\nu}$ is the energy emitted in neutrinos. We shall assume $E_{\nu} \approx 100 E_{\gamma}[2,11]$. The total number of neutrinos emitted is given by $N_{\nu}=E_{\nu} /\left\langle E_{\nu}\right\rangle$, where $\left\langle E_{\nu}\right\rangle=3.15 T_{\nu}$ is the average energy of the neutrinos, assuming a Fermi-Dirac distribution. From this we obtain

$$
N_{\nu}=7 \times 10^{57}\left(\frac{E_{\nu}}{10^{54} \mathrm{ergs}}\right)^{3 / 4}\left(\frac{R}{100 \mathrm{~km}}\right)^{3 / 4} .
$$

The neutrino flux at Earth is

$$
\Phi_{\nu}=\frac{N_{\nu}}{4 \pi D^{2}} \frac{4 \pi}{\Omega_{\text {beam }}}
$$

where $D$ is the distance of the GRB given by Eq.12 and $\Omega_{\text {beam }}$ is the beaming solid angle of the GRB corresponding to a cone of opening angle $\theta_{\text {beam }}$, which we assume to be of order $\theta_{\text {beam }} \sim 1^{\circ}$ [24]. The total number of events observed at the detector by $\bar{\nu}_{e}$ capture on protons is given by

$$
N=N_{p} \int \phi(E) \sigma(E) d E
$$

where the $\bar{\nu}_{e}$ flux $\phi(E)=\frac{1}{6} \Phi_{\nu} f_{F D}(E), f_{F D}(E)$ being the Fermi-Dirac distribution function and we assume that the total neutrino flux $\Phi_{\nu}$ is evenly distributed in all the six species. The reaction cross-section $\sigma(E)$ for $\bar{\nu}_{e}+p \rightarrow n+e^{+}$is given by

$$
\sigma(E)=0.94 \times 10^{-43}(E-Q)\left((E-Q)^{2}-m_{e}^{2}\right)^{1 / 2} \mathrm{~cm}^{2}
$$

where $Q=1.293 \mathrm{MeV}$ is the neutron-proton mass difference and $m_{e}$ is the electron mass. $N_{p}=$ $\frac{2}{18} N_{A} M_{D}$ is the number of free protons in water, where $N_{A}$ is the Avogadro's number and $M_{D}$ is the fiducial mass of the detector. The integration in Eq.22 is done over observed neutrino energies. In Figure 3 we show the number of events expected in a one megaton water Cerenkov detector from thermal neutrinos produced in a GRB, which is beamed towards us with a beaming angle of $1^{\circ}$ [24]. The number of expected events are seen to be large for the GRBs with low redshift.

In Figure 4 we show the time delay $\Delta t$ in seconds as a function of $\epsilon_{0}=m c^{2} / E_{0}$ for redshift $z=0.01,0.02,0.05,0.1,0.2,0.5,1,10$, for the cosmological constant model. In this figure we assume that the neutrino energy satisfies $E_{0}<E_{0}^{\prime}$ so that the effects of quantum gravity can be neglected and hence we can set $\epsilon_{0}^{\prime}=0$. For a fixed redshift the time delay increases as $\epsilon_{0}^{2}=m^{2} c^{4} / E_{0}^{2}$ giving larger time delays for more massive neutrinos and lower observed energies, as seen in Figure 2 . We are interested in measuring the smallest neutrino masses, which for a fixed $\epsilon_{0}$ corresponds to the smallest observed energies $E_{0}$ and the smallest time delays. Since the neutrinos are released from the GRB in bursts on the time scale of milliseconds, the smallest time delays that are meaningful will also be milliseconds and from the figure this corresponds to $\epsilon_{0}=m c^{2} / E_{0} \sim 10^{-10}$. For the lowest detectable energy neutrinos of $E_{0} \sim 10 \mathrm{MeV}, \epsilon_{0} \sim 10^{-10}$ corresponds to neutrino masses of 


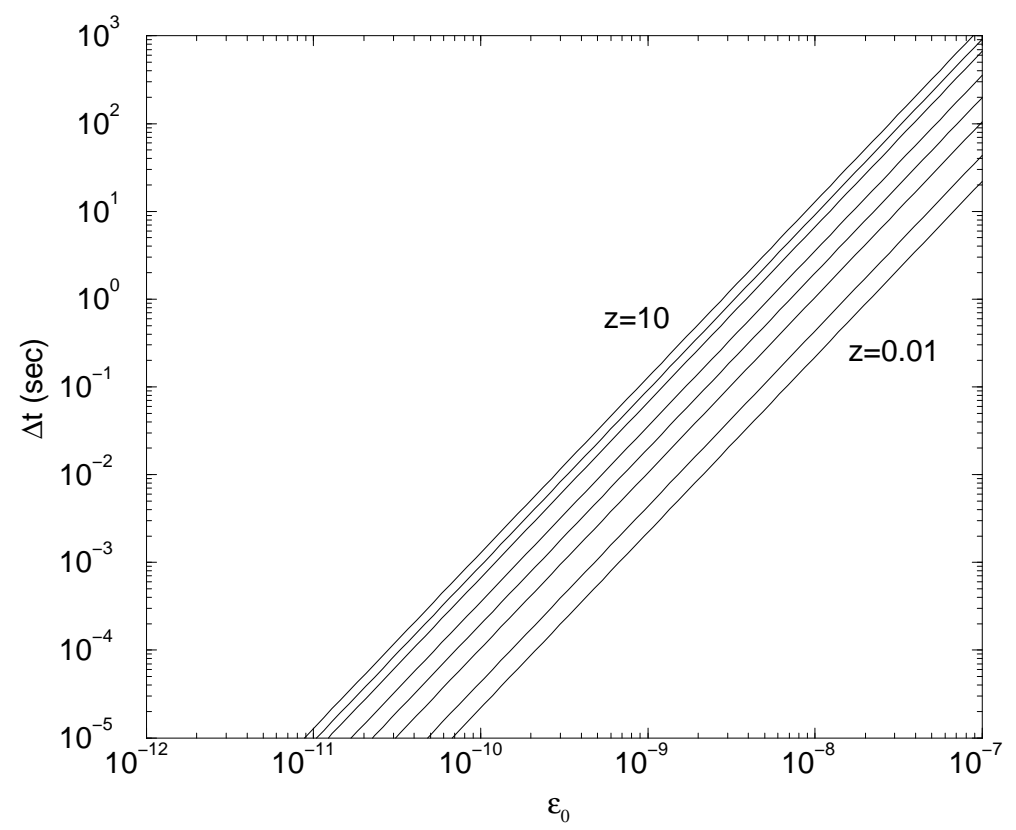

Figure 4: The time delay $\Delta t$ in seconds as a function of $\epsilon_{0}=m c^{2} / E_{0}$ for redshift $z=$ $0.01,0.02,0.05,0.1,0.2,0.5,1,10$, assuming the cosmological constant model and taking $\epsilon_{0}^{\prime}=0$.

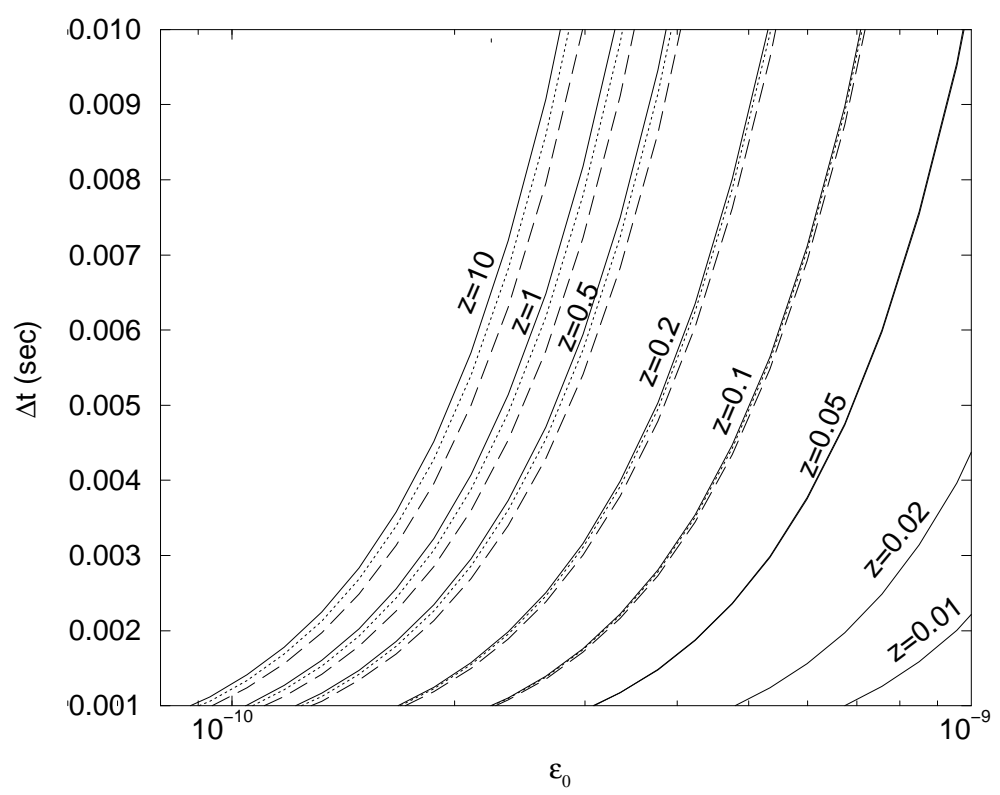

Figure 5: Variation in time delay $\Delta t$ in the millisecond region with $\epsilon_{0}=m c^{2} / E_{0}$ for red shift of $z=0.01,0.02,0.05,0.1,0.2,0.5,1,10$, for the three different cosmological models (taking $\epsilon_{0}^{\prime}=0$ ): CONST (solid), SUGRA quintessence (dots), INV quintessence (dashes). 
$m \sim 10^{-3} \mathrm{eV}$. In order to discuss the limits on neutrino masses in more detail we must focus on the millisecond region of this plot.

In Figure 5 we show a blow-up of Figure 4 in the important millisecond region. We have also included the effect of different cosmological models in this figure, which can be important for high redshifts. From Figure 5 we see that time delays of $10^{-3}$ s correspond to $\epsilon_{0}=7 \times 10^{-10}-8 \times 10^{-11}$, over a range of redshift $z=0.01-10$. For the lowest energy neutrinos of $E_{0} \sim 10 \mathrm{MeV}$ this range of $\epsilon_{0}$ corresponds to a range of neutrino masses of $m=7 \times 10^{-3}-8 \times 10^{-4} \mathrm{eV}$. Although the smallest neutrino masses are associated with the highest redshifts the number of events expected in the detector falls sharply with increasing redshift. Since $I_{-1}$ is not very sensitive to $z$ beyond $z \sim 1$, the fall in the number of events with distance is more acute than the rise in the time delay, and hence GRBs at lower values of redshift would be better suited for determination of the neutrino mass.

In addition to being sensitive to the absolute masses of all the three neutrino states, GRB neutrinos have the potential to probe the mass hierarchy as well. Though from solar neutrino data we know that the sign of $\Delta m_{21}^{2} \equiv \Delta m_{\odot}^{2}$ is positive $[22,23]\left(\Delta m_{i j}^{2}=m_{i}^{2}-m_{j}^{2}\right)$, there is still an ambiguity in the sign of $\Delta m_{32}^{2} \equiv \Delta m_{a t m}^{2}$. It would be hard to determine the sign of $\Delta m_{32}^{2}$ in any of the current and planned long baseline oscillation experiments and only a neutrino factory would be able to resolve this ambiguity. The delay in arrival times for the neutrinos can in principle be used to determine the neutrino mass hierarchy. Neutrinos arrive in three bunches corresponding to the three mass eigenstates. The neutrinos are detected via the electron antineutrino reaction, so for each mass eigenstate we must multiply the detection rate by the probability $\left|U_{e i}\right|^{2}$ that the mass eigenstate corresponding to the mass $m_{i}$ contains an electron antineutrino. Since $\left|U_{e 3}\right|^{2}$ is small $m_{3}$ will have the smallest component of electron antineutrino and hence smallest number of events at the detector. In practice the states with mass $m_{1}$ and $m_{2}$ may be most easily detectable since $\left|U_{e 1}\right|^{2}$ and $\left|U_{e 2}\right|^{2}$ are not too small. The heaviest mass eigenstate will arrive last and have the largest time delay. In hierarchical models the heaviest mass is $m_{3}$ while in models with inverted mass hierarchy $m_{3}$ is the lightest. So depending on whether fewer events are detected for the neutrinos which arrive last (earliest) it would in principle be possible to conclude that the mass hierarchy is normal (inverted).

\subsection{Quantum Gravity Limits from High Energy Neutrinos}

The dispersion of velocity due to quantum gravity effects leading to delay in arrival times of the neutrinos is most significant at the higher energy end of the GRB neutrino spectrum, as evident from Figure 2. In fact for a given neutrino mass $m$, all neutrinos with energy $E_{0}>E_{0}^{\prime}$ can be used to put limits on the scale of quantum gravity $E_{Q G}$. Since neutrinos with a wide range of energies from $1-10^{9} \mathrm{GeV}$ are expected to be produced in GRB fireball $[6,7,8,9,10]$, the detection of their time delays is the most powerful method of studying/constraining models which predict dispersion relations given by Eq.1.

In this section we therefore focus on neutrinos with energy $E_{0}>E_{0}^{\prime}$ in order to set limits on the quantum gravity scale. Figure 6 shows the time delay expected as a function of $\epsilon_{0}^{\prime}=E_{0} / E_{Q G}$ for redshifts of $0.01,0.02,0.05,0.1,0.2,0.5,1$ and 10, assuming the cosmological constant model and also that $E_{0}>E_{0}^{\prime}$ so that we can set $\epsilon_{0}=0$. As also seen in Figure 2 the time delays in Figure 


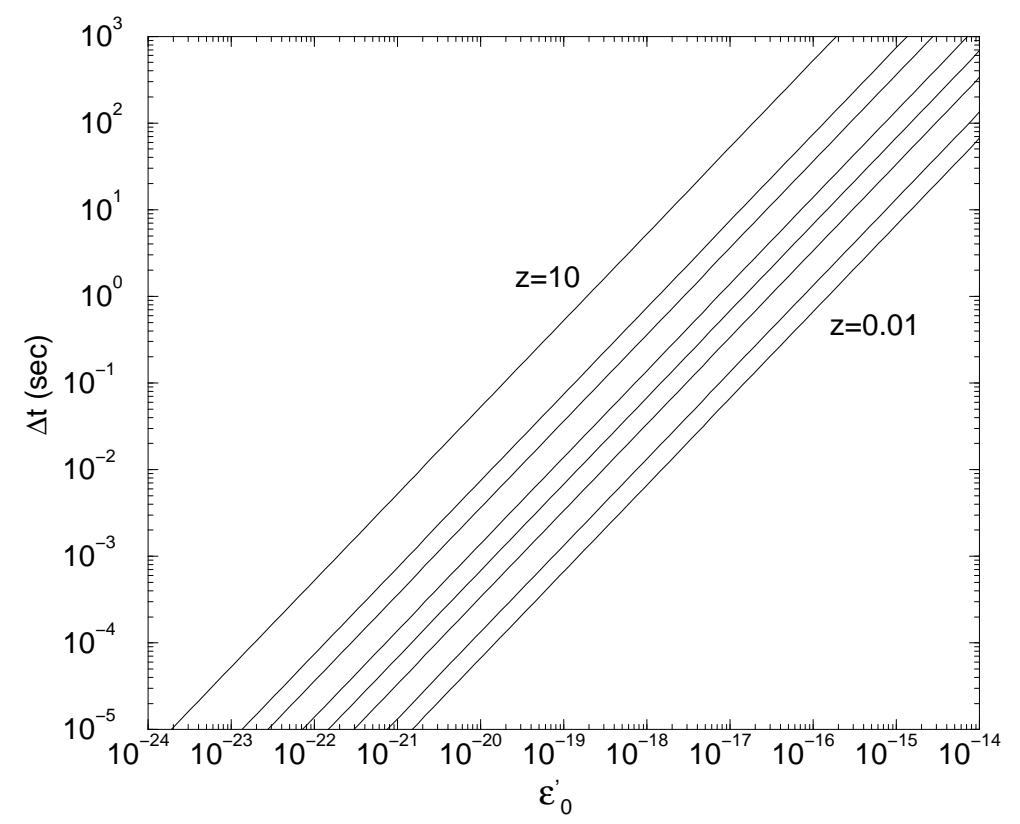

Figure 6: The time delay $\Delta t$ in seconds as a function of $\epsilon_{0}^{\prime}=E_{0} / E_{Q G}$ for redshift $z=$ $0.01,0.02,0.05,0.1,0.2,0.5,1,10$, assuming the cosmological constant model and taking $\epsilon_{0}=0$.

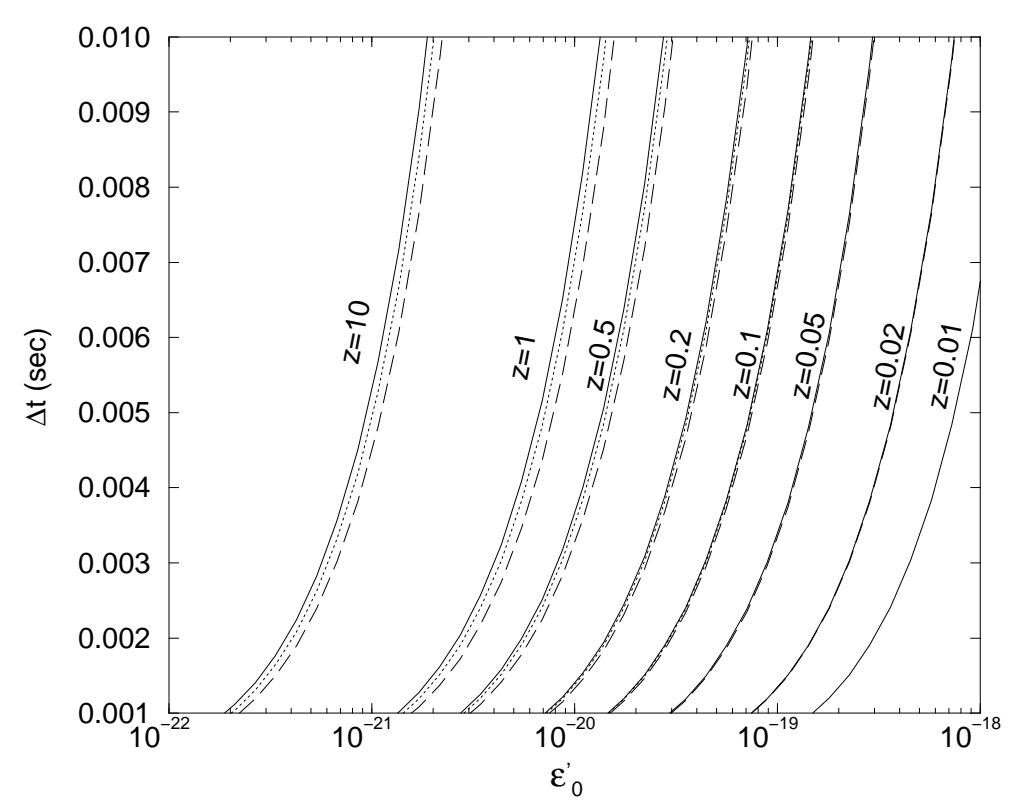

Figure 7: Variation in the quantum gravity induced time delay $\Delta t$ in the millisecond region with $\epsilon_{0}^{\prime}=E_{0} / E_{Q G}$ for red shift of $z=0.01,0.02,0.05,0.1,0.2,0.5,1,10$, for the three different cosmological models (taking $\epsilon_{0}=0$ ): CONST (solid), SUGRA quintessence (dots), INV quintessence (dashes). 
6 increase linearly with energy and hence with $\epsilon_{0}^{\prime}$. The time delay also increases significantly with the redshift $z$ as $I_{-1}$ has a sharp $z$ dependence. Since we want to restrict ourselves to minimum time delays of a millisecond, which corresponds to the variability time of the GRBs, we show in Figure 7 the blow up of the region with $\Delta t \sim 10^{-3}$ seconds. We also show the effect of the different cosmological models on time delays on the same plot. For $z=0.01-10$ the range of $\epsilon_{0}^{\prime}$ which would give $\Delta t \leq 10^{-3}$ seconds is $\epsilon_{0}^{\prime} \leq 1.5 \times 10^{-19}-2 \times 10^{-22}$. For the highest energy neutrinos with $E_{0} \sim 10^{9} \mathrm{GeV}$ expected from the GRB, this would translate into $E_{Q G} \geq 6 \times 10^{28}-5 \times 10^{30}$ $\mathrm{GeV}$. This should be compared with the bounds set by the time delays of the photons observed from GRBs [13] where the tightest limit obtained from the highest energy observed events is $E_{Q G} \geq 8.3 \times 10^{16} \mathrm{GeV}[15]$. In the extreme ultra-relativistic regime the effect of neutrino mass is negligible and the dispersion of the neutrinos in the quantum space-time foam would be almost the same as that of photons, the latter having the advantage of being easier to detect. However ultra-high energy photons are subject to the GZK cut-off and beyond that for better bounds on $E_{Q G}$ one can use ultra-high energy neutrinos. Bounds on $E_{Q G}$ from non-observation of dispersion effects of the quantum space-time foam in neutrino oscillations experiments is slightly stronger with $E_{Q G} \gtrsim 10^{22} \mathrm{GeV}[25]$. This upper limit may be improved in the forthcoming long baseline experiments using neutrino superbeams. But it should be noted that this effect will show up in neutrino oscillations only if the dispersion due to quantum gravity is flavor dependent. However the method using time delays will be sensitive even if the effect is flavor independent. Thus the observation of time delays of GRB neutrinos is the most promising way of phenomenologically testing quantum gravity.

\subsection{Dark Energy Limits from High Redshift Events}

Different dark energy models give different values for the Hubble constant $H(z)$ at a given redshift. This can have significant impact on the time delays. Since the difference in $H(z)$ is maximum at the highest redshifts, we have to look at time delays of neutrinos coming from the farthest GRB events to probe dark energy. Since from Figure 1 we see that $I_{-1}$ has a much better sensitivity to dark energy than $I_{2}$, time delays arising from quantum gravity would be a better probe for the different cosmological models. Hence it is the ultra high energy neutrinos which would be potentially more sensitive. In Figure 8 we show a further blow-up of the time delay plot due to quantum gravity effects, with $\Delta t$ between $5 \times 10^{-3}-10^{-2}$ seconds and $\epsilon_{0}^{\prime}$ between $10^{-21}-10^{-20}$. We show the plots for $z=1,2,4,6$ and 10. The three different line types correspond to the three different models for the dark energy that we have considered in this paper. The time delay obtained depends on the redshift as well as the cosmological model. For $z>3$ the time delays for different redshifts and different cosmologies begin to overlap. This ambiguity can be resolved by using smaller redshift events to determine $E_{Q G}$, since for such events the effect of dark energy is reduced. Once $E_{Q G}$ is determined then one can use the high redshift GRB neutrinos to probe dark energy. In order to successfully probe the correct cosmological model it is important that the redshift of the distant GRBs can be determined accurately enough from their afterglow and that the scale of $E_{Q G}$ can be ascertained well enough by looking at the time delay for neutrinos from GRB from lower redshifts. If this can be achieved then information on $\Delta t$ for neutrinos coming from the very distant sources can be used with information on $z$ and the scale of $E_{Q G}$ to probe 


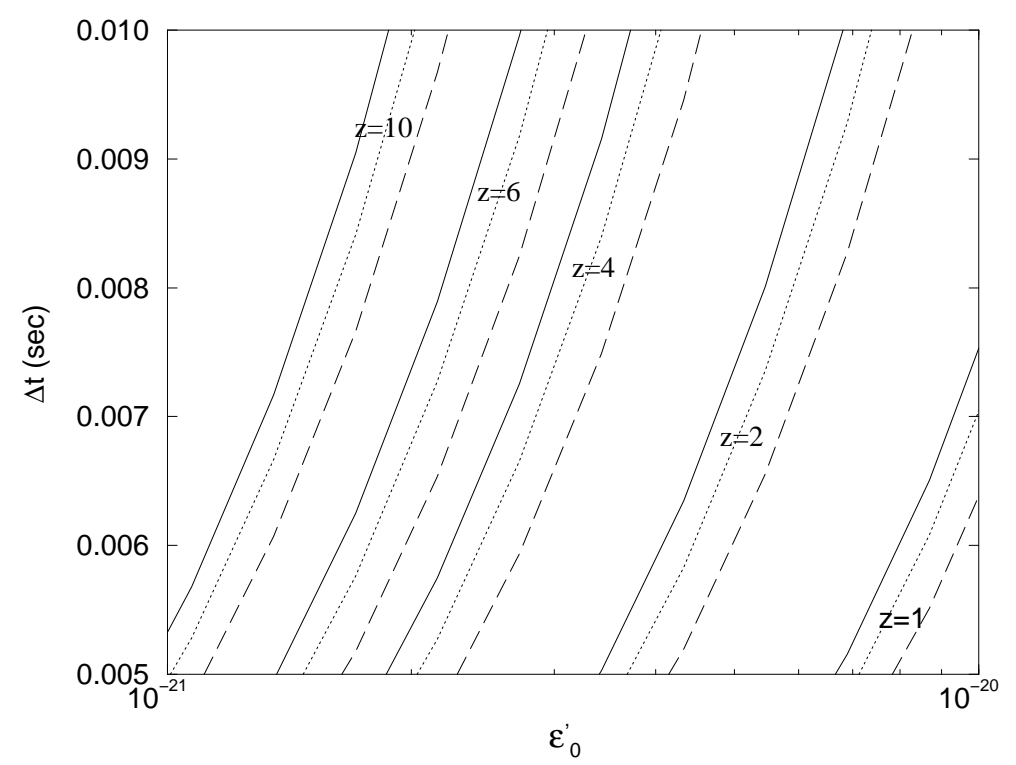

Figure 8: Variation in the quantum gravity induced time delay $\Delta t$ in the millisecond region with $\epsilon_{0}^{\prime}=E_{0} / E_{Q G}$ for high red shifts of $z=1,2,4,6,10$ (from right to left) for the three different cosmological models (taking $\epsilon_{0}=0$ ): CONST (solid), SUGRA quintessence (dots), INV quintessence (dashes).

the correct cosmological model.

\section{Discussion and Conclusion}

In this paper we have calculated the time delays of neutrinos emitted in gamma ray bursts due to the effects of neutrino mass and quantum gravity. Our results are based on the formula for $\Delta t$ in Eq.9, using the Hubble constant for a flat universe calculated using Eq.13 for different dark energy models. This formalism correctly takes into account the time dependence of the Hubble constant due to matter and dark energy, and can change the naive results in the literature by more than $100 \%$ for $z>1$. We have shown that the effects of neutrino mass, quantum gravity and dark energy may be disentangled by using low energy neutrinos to study neutrino mass, high energy neutrinos to study quantum gravity, and large redshifts to study dark energy. From low energy neutrinos one may obtain direct limits on neutrino masses of order $10^{-3} \mathrm{eV}$, and distinguish a neutrino mass hierarchy from an inverted mass hierarchy. From ultra-high energy neutrinos the sensitivity to the scale of quantum gravity can be pushed up to $E_{Q G} \sim 5 \times 10^{30} \mathrm{GeV}$. By studying neutrinos from GRBs at large redshifts a cosmological constant could be distinguished from quintessence.

For convenience we have calculated all time delays with respect to a hypothetical low energy photon, assumed to be emitted at the same time from a point source as the neutrino of a given energy. We emphasise that what is important in practical search strategies is not these time delays themselves, which will be unmeasurable due to the uncertainties in the emission characteristics of low energy photons, but rather the comparison of time delays between neutrinos of either the 
same energy, or between high energy neutrinos of two different energies. As already mentioned, if the neutrinos are hierarchical in mass then neutrinos of the same energy will arrive at the detector in three bunches, and we can then compare the arrival times of the different neutrino mass eigenstates and put limits on the neutrino mass, as in the case of supernova neutrinos [19] but with sensitivities better by orders of magnitude. For constraining models of quantum gravity and dark energy one can use arrival times of the ultra-high energy neutrinos, and can compare the arrival times of the high energy neutrinos of different energies.

\section{Acknowledgement}

We particularly acknowledge very helpful discussions with Tom Marsh. We also wish to thank

Juan Garcia-Bellido, Christian Kaiser, Ian McHardy, Tim Morris and Graham Shore for helpful discussions, and Francis Halzen for useful communications.

\section{References}

[1] For an introductory review of GRBs see for example G.Ghisellini, astro-ph/0111584.

[2] F. Halzen and G. Jaczko, Phys. Rev. D 54 (1996) 2779.

[3] J. Alvarez-Muniz, F. Halzen and D. W. Hooper, Phys. Rev. D 62 (2000) 093015 [arXiv:astro$\mathrm{ph} / 0006027]$.

[4] F. Halzen and D. W. Hooper, Astrophys. J. 527 (1999) L93 [arXiv:astro-ph/9908138].

[5] N. Gupta, Phys. Rev. D 65 (2002) 113005 [arXiv:astro-ph/0201509].

[6] E. Waxman and J. N. Bahcall, Phys. Rev. Lett. 78 (1997) 2292 [arXiv:astro-ph/9701231].

[7] E. Waxman and J. N. Bahcall, Phys. Rev. D 59 (1999) 023002 [arXiv:hep-ph/9807282].

[8] J. N. Bahcall and E. Waxman, Phys. Rev. D 64 (2001) 023002 [arXiv:hep-ph/9902383].

[9] E. Waxman and J. N. Bahcall, Astrophys. J. 541 (2000) 707 [arXiv:hep-ph/9909286].

[10] J. N. Bahcall and P. Meszaros, Phys. Rev. Lett. 85, 1362 (2000) [arXiv:hep-ph/0004019].

[11] F. Halzen and D. Hooper, arXiv:astro-ph/0204527. 
[12] A. D. Rujula, arXiv:astro-ph/0207033.

[13] G. Amelino-Camelia, J. R. Ellis, N. E. Mavromatos, D. V. Nanopoulos and S. Sarkar, Nature 393 (1998) 763 [arXiv:astro-ph/9712103].

[14] S. D. Biller et al., Phys. Rev. Lett. 83 (1999) 2108 [arXiv:gr-qc/9810044].

[15] B. E. Schaefer, arXiv:astro-ph/9810479.

[16] J. R. Ellis, K. Farakos, N. E. Mavromatos, V. A. Mitsou and D. V. Nanopoulos, Astrophys. J. 535 (2000) 139 [arXiv:astro-ph/9907340].

[17] J. Alfaro, H. A. Morales-Tecotl and L. F. Urrutia, Phys. Rev. Lett. 84 (2000) 2318 [arXiv:grqc/9909079].

[18] J. R. Ellis, N. E. Mavromatos, D. V. Nanopoulos and G. Volkov, Gen. Rel. Grav. 32 (2000) 1777 [arXiv:gr-qc/9911055].

[19] J. F. Beacom and P. Vogel, Phys. Rev. D 58, 093012 (1998) [arXiv:hep-ph/9806311].

[20] P. S. Corasaniti and E. J. Copeland, arXiv:astro-ph/0205544.

[21] Y. Fukuda et al. [Super-Kamiokande Collaboration], Phys. Rev. Lett. 81 (1998) 1562 [arXiv:hep-ex/9807003].

[22] A. Bandyopadhyay, S. Choubey, S. Goswami and D. P. Roy, Phys. Lett. B 540 (2002) 14 [arXiv:hep-ph/0204286].

[23] J. N. Bahcall, M. C. Gonzalez-Garcia and C. Pena-Garay, arXiv:hep-ph/0204314.

[24] D.A Frail et al, Astrophysical Journal 562:L55-L58 (2001)

[25] R. Brustein, D. Eichler and S. Foffa, Phys. Rev. D 65 (2002) 105006 [arXiv:hep-ph/0106309]. 